\title{
Gamma-ray irradiation promotes premature meiosis of spontaneously differentiating testis-ova in the testis of p53-deficient medaka (Oryzias latipes)
}

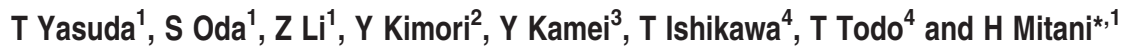

In this study, the roles of $\mathrm{p} 53$ in impaired spermatogenic male germ cells of p53-deficient medaka were investigated by analyzing histological changes, and gene expressions of 42Sp50, Oct 4 and vitellogenin (VTG2) by RT-PCR or in situ hybridization in the testes. We found that a small number of oocyte-like cells (testis-ova) differentiated spontaneously in the cysts of type $A$ and early type B spermatogonia in the p53-deficient testes, in contrast to the wild-type (wt) testes in which testis-ova were never found. Furthermore, ionizing radiation (IR) irradiation increased the number of testis-ova in p53-deficient testes, increased testis-ova size and proceeded up to the zygotene or pachytene stages of premature meiosis within 14 days after irradiation. However, 28 days after irradiation, almost all the testis-ova were eliminated presumably by p53-independent apoptosis, and spermatogenesis was restored completely. In the wt testis, IR never induced testis-ova differentiation. This is the first study to demonstrate the pivotal role of the p53 gene in the elimination of spontaneous testis-ova in testes, and that p53 is not indispensable for the restoration of spermatogenesis in the impaired testes in which cell cycle regulation is disturbed by IR irradiation.

Cell Death and Disease (2012) 3, e395; doi:10.1038/cddis.2012.133; published online 4 October 2012

Subject Category: Cancer

Germ cells are responsible for transmitting genetic information from generation to generation. If mutations occur in male germ cells, they are transmitted to offspring and may induce malformations or diseases. ${ }^{1,2}$ The tumor suppressor p53 is considered a guardian of genome integrity, regulating the cell cycle in proliferating cells to repair genomic lesions, and removing severely injured cells by apoptosis in vivo. ${ }^{3}$ In this study, to clarify the roles of p53 when spermatogenesis is disrupted by ionizing radiation (IR) irradiation, we examined the histological changes in the irradiated testes of wild-type (wt) and p53-deficient mutant fish of medaka (Oryzias latipes) obtained by the TILLING (targeting-induced local lesions in genomes) method. ${ }^{4}$

The medaka is a unique vertebrate model for investigating the effects of IR on germ cells, because of the simple architecture of its testis, and the fact that the specific locus method can be used with precise accuracy to evaluate mutation rates in germ cells. ${ }^{5,6}$ Furthermore, it has been demonstrated that mutation rates in medaka germ cells are almost equal to those of mice..$^{5,6}$ The mature testes of medaka consist of many cysts containing differentiating spermatogenic cells (spermatogonia, spermatocytes, spermatids and spermatozoa), and spermatogonial stem cells
(SSC), each wrapped by one Sertoli cell, forming a niche. ${ }^{7}$ The ovaries and testes are composed of structurally similar units (fundamental units) that comprise a layer of supporting cells surrounding the germ cells. ${ }^{8}$ As cells in a cyst differentiate synchronously, and all of the cells in a cyst are at the same stage of spermatogenesis, it is possible to identify the different spermatogenic stages by histological examination more easily and precisely than in mammals. ${ }^{9}$ In previous studies, it was reported that IR-induced genomic lesions in germ cells are eliminated in one of the two ways: either by DNA repair and apoptosis, or by the reset of spermatogenesis; surviving irradiated spermatogenic cells, except for the SSC, are prematurely removed from the testis by acceleration of spermatogenesis. ${ }^{10,11}$ There are reports that spontaneous sex reversal occurs in the gonads of newts and chicken embryos. ${ }^{12,13}$ The medaka has an $X Y$ sex determination system, whereby the dominant gene DMY/dmrt1bY on the $Y$ chromosome determines male differentiation. ${ }^{14-16}$ To date, the spontaneous differentiation of oocyte like cells (testis-ova) has not been reported in the medaka. Various methods, such as steroid sex hormone or hormone mimetic chemical treatments, ${ }^{17}$ and IR treatment can enhance the artificial induction of ova-like cells in medaka testes. ${ }^{18}$

\footnotetext{
${ }^{1}$ Department of Integrated Biosciences, Graduate School of Frontier Sciences, The University of Tokyo, Bioscience Bldg. 102, Kashiwa, Chiba 277-8562, Japan; ${ }^{2}$ Imaging Science Division, Center for Novel Science Initiatives, National Institutes of Natural Sciences, Toranomon 4-3-13, Minato-ku, Tokyo 105-0001, Japan; ${ }^{3}$ Spectrography and Bioimaging Facility, NIBB Core Facilities, National Institute for Basic Biology, Nishigo-naka 38, Myodaiij, Okazaki, 444-8585 Aichi, Japan and ${ }^{4}$ Department of Radiation Biology and Medical Genetics, Graduate School of Medicine, Osaka University, B4, 2-2 Yamadaoka, Suita, Osaka 565-0871, Japan ${ }^{*}$ Corresponding author: H Mitani, Department of Integrated Biosciences, Graduate School of Frontier Sciences, The University of Tokyo, Bioscience Bldg. 102, Kashiwa, Chiba 277-8562, Japan. Tel: +81 43 471363670; Fax: +81 43 471363669; E-mail: mitani@k.u-tokyo.ac.jp

Keywords: sex differentiation; testis-0va; p53; irradiation; apoptosis; medaka

Abbreviations: IR, ionizing radiation; wt, wild type; TILLING, targeting-induced local lesions in genomes; SSC, spermatogonial stem cells; oocyte-like cells, testis-ova; H\&E, hematoxylin and eosin; ISH, in situ hybridization; PGCs, primordial germ cells; VTG2, vitellogenin; fancl, Fanconi anemia complementation group L

Received 19.3.12; revised 09.7.12; accepted 10.7.12; Edited by A Stephanou
} 
In this study, we demonstrated that testis-ova differentiate spontaneously from spermatogonia in p53-deficient medaka, and IR irradiation promotes their differentiation and development. Nearly all of these testis-ova were eliminated and spermatogenesis was almost completely restored within 28 days of irradiation. Based on these results, the p53dependent and p53-independent pathways in the maintenance of genomic integrity in spermatogenesis are revealed. This is the first report of the p53 gene having a pivotal role in the elimination of spontaneously differentiating testis-ova in testes, and in response to IR irradiation.

\section{Results}

Spontaneous testis-ova reside in control p53-deficient medaka testes. All p53-deficient male medaka are as fertile as wt male medaka and have a normal male-type external appearance such as dorsal and anal fins (arrows and arrowheads in Figures $1 \mathrm{a}$ and $\mathrm{b}$ ). Both testes of wt and p53-deficient medaka males are bilobate organs that consist of a large number of cysts filled with synchronously
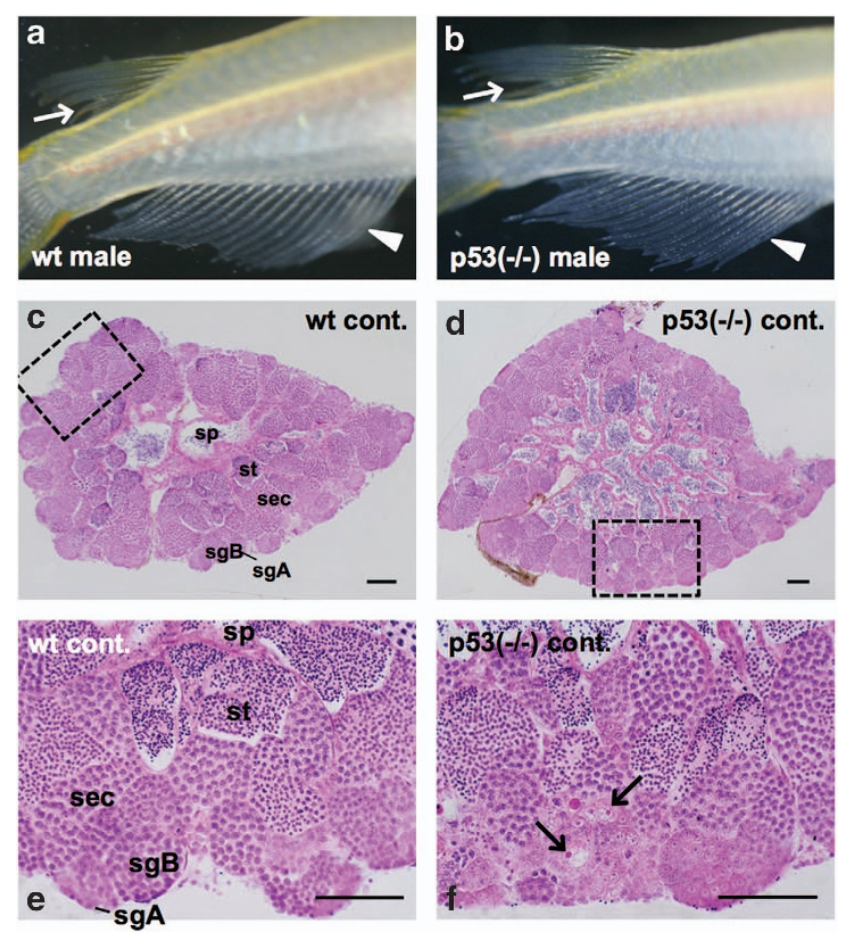

Figure 1 Testis-ova were differentiating spontaneously in the non-irradiated p53 $(-/-)$ testis. Representative images of the secondary sex characteristics of p53 $(-/-)$ medaka adults that are genetically male (b), showing typical male-type external appearance of wt male medaka (a) such as rougher edges of dorsal fins (arrows in $\mathbf{a}$ and $\mathbf{b}$ ) and sharply long anal fins (arrowheads in $\mathbf{a}$ and $\mathbf{b}$ ). H\&E-stained sections of non-irradiated testes of wt and p53( $-/-$ ) fishes at 6 months old. (c) wt testis. (d) p53( - / - ) testis. (e) Enlarged view of the boxed area in (c). (f) Enlarged view of the boxed area in (d) showing a small number of characteristic cells positioned in the cysts with type A or B spermatogonia, of which the nucleolus was strongly $\mathrm{H} \& E$-stained and the nucleus was faintly stained (arrows in f). sgA, type $A$ spermatogonia; sgB, type B spermatogonia; sec, spermatocytes; st, spermatids; sp, spermatozoa. Scale bars represent $50 \mu \mathrm{m}$ differentiating spermatogenic cells: type A and type B spermatogonia, spermatocytes, spermatids and spermatozoa (sp) (Figures 1c and d). This indicates that a lack of p53 does not affect the general architecture of the testis. However, we found that all testes of p53-deficient medaka males contained a small number of characteristic cells in the cysts filled with type A or early type B spermatogonia, which were clearly distinguishable from the other spermatogenic cells because of their strongly hematoxylin and eosin (H\&E)stained nucleolus, and very faintly H\&E-stained nucleus (arrows in Figure 1f). The appearance of these cells suggests that they are in the testis (a result confirmed by molecular analyses. See the later paragraph), hence we designated them as testis-ova. Testis-ova were found only in the testes of p53-deficient medaka, and no testis-ova were present in wt Hd-rR testes (Figures 1e and 3).

Morphological changes of testis-ova during 1 month after $\gamma$-ray (5Gy) irradiation in p53-deficient medaka testes. One day after $5 \mathrm{~Gy} \gamma$-ray irradiation, there were no obvious histological changes in H\&E-stained sections of p53deficient medaka testes (Figures $2 a$ and $b$ ). Three days after irradiation, pyknotic cells that had characteristic staining with $\mathrm{H} \& \mathrm{E}$ appeared (open arrowheads in Figure 2c), and the number of testis-ova started to increase in the cysts of type A or early type B spermatogonia throughout the whole area (arrows in Figure 2c). Seven days after irradiation, the number of testisova increased in the cysts of type $A$ or early type $B$ spermatogonia and spermatocytes (Figure 2d). Electron microscopy revealed the increased number of testis-ova at synchronous stage of development in each cysts (arrowheads in Figure 4b). Electron microscopy also confirmed that these testis-ova were differentiating at various stages of meiotic prophase. Almost all of the testis-ova had distinct short and thick chromatin (arrows with $c$ in Figures 4b and e) and were at the leptotene stage, while the others had chromosome structures with synaptonemal complexes (arrows in Figure 4f) and were at the zygotene or pachytene stages. Type A spermatogonia in the non-irradiated wt testes can be distinguished from other spermatogenic cells because of their characteristic germinal dense bodies that were so called nuage in the cytoplasm (arrowhead with $n$ in Figure 4c) that are closely associated with large aggregations of mitochondria (arrows with $\mathrm{m}$ in Figure 4c). Although the ultrastructure of the cytoplasm in testis-ova at meiotic prophase stages (Figure 4d) was generally similar to that of type A spermatogonia (Figure 4c), characteristic features of the cytoplasm in testis-ova were the increased number, and more electron-dense matrix of the mitochondria than those in type A spermatogonia (arrows with $\mathrm{m}$ in Figures $4 \mathrm{c}$ and $\mathrm{d}$ ). Moreover, remarkably larger and strongly electron-dense nucleolus (open arrowhead in Figure 4d) than that of nonirradiated wt spermatogonia (open arrowhead in Figure 4c) was detected as characteristic feature of testis-ova. These ultrastructural differences between spermatogonia and testis-ova are consistent with the histological differences of germ cells of males and females during sexual differentiation. ${ }^{19}$

Strongly H\&E-stained pyknotic cells were observed up to 14 days after irradiation (open arrowheads in Figures $2 \mathrm{~g}$ and $\mathrm{h}$ ). 

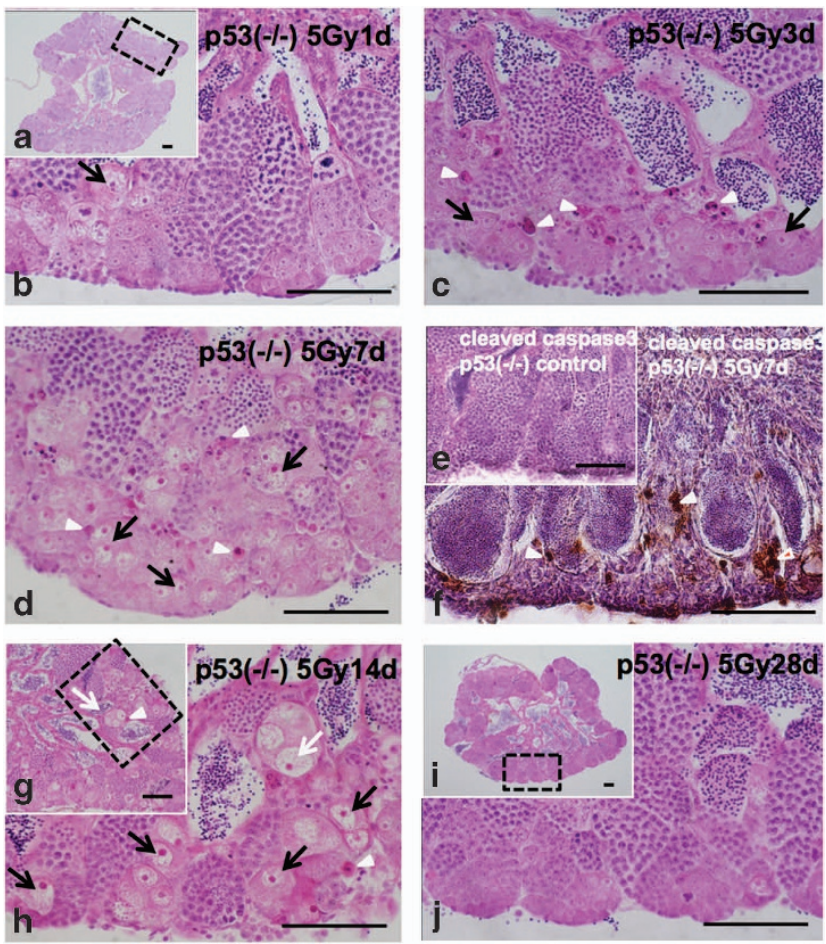

Figure 2 Histological changes of testis-ova in a p53(-/ - ) testis after 5 Gy $\gamma$-ray irradiation. Histological changes of testis-ova in a p53 $(-/-)$ testis during 28 days (1 month) after irradiation with 5 Gy of $\gamma$-rays. The arrowheads in $\mathbf{c}, \mathbf{d}, \mathbf{f}, \mathbf{g}$ and $\mathbf{h}$ indicate the H\&E-stained pyknotic cells, and arrows in $\mathbf{c}, \mathbf{d}, \mathbf{g}$ and $\mathbf{h}$ indicate the testis-ova. (a) H\&E-stained section 1 day after irradiation. (b) Enlarged view of the boxed area in (a), showing that no apparent histological changes. (c) H\&E-stained section 3 days after irradiation. (d) H\&E-stained section 7 days after irradiation. H\&E-stained pyknotic cells (arrowheads in d) that were positive in immunostaining against anticleaved caspase 3 (arrowheads in $\mathbf{f}$ ) in contrast to no positive signals in control p53 $(-/-)$ medaka (e), showing that these pyknotic cells are apoptotic. Testis-ova (arrows in d) increased extensively in the cysts of type A and early type B spermatogonia. (e) Immunostaining against anticleaved caspase3 in control p53 $(-/-)$ medaka testis. (g) H\&E-stained section 14 days after irradiation. (h) Enlarged view of the boxed area in (g), showing that H\&E-stained pyknotic cells (arrowheads in $\mathbf{d}, \mathbf{f}, \mathbf{g}$ and $\mathbf{h}$ ) were observed continuously, and the larger size of testis-ova when compared with those 7 days after irradiation were observed (open arrows in $\mathbf{g}$ and $\mathbf{h}$ ). (i) H\&E-stained section of testis 28 days after irradiation. (j) Enlarged view of the boxed area in (i), showing that almost of all testis-ova had disappeared from the testis. Scale bars represent $50 \mu \mathrm{m}$

Testes of p53-deficient medaka males 7 days after 5 Gy $\gamma$-ray irradiation were immunostained with an antibody against activated caspase-3, which is an early marker for apoptotic cells. The H\&E-stained pyknotic cells, including those of the testis-ova, were immuno-positive (open arrowheads in Figure 2f), indicating that they were apoptotic. Electron microscopic observations of testes 7 days after irradiation revealed that condensed nuclei of apoptotic cells were positioned in the same cyst with testis-ova (arrowheads in Figure 4e) and these apoptotic cells were phagocytosed in enlarged Sertoli cells (arrowheads in Supplementary Figure S1C-E). In the mass of spermiated mature spermatozoa, apoptotic testis-ova were sometimes observed (arrowhead in Supplementary Figure S1F). The number of spermatocytes decreased (Supplementary Table S1), which was consistent with $\gamma$-ray-irradiated wt testes reported in previous papers, ${ }^{10,11}$ suggesting that acceleration

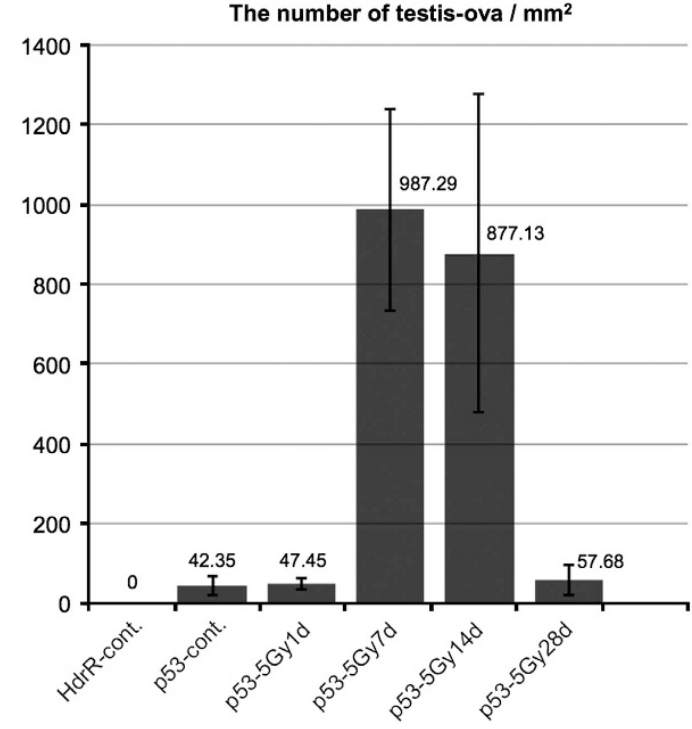

Figure 3 Time course of the number of testis-ova in $\gamma$-ray-irradiated p53 $(-/-)$ testes. The number of testis-ova were counted on the histological section at the center of testes in $\gamma$-ray-irradiated testes of p53-deficient medaka at 1 day, 7 days, 14 days and 28 days after irradiation, and in a non-irradiated testis of both wt and p53deficient medaka testes ( $n=3$ for all time points). The error bars represent s.d.'s of means

of spermatogenesis also occurred in p53-deficient medaka testes.

Fourteen days after 1 or 5 Gy $\gamma$-ray irradiation, histological observations showed that the sizes of H\&E-stained testis-ova had increased diameters ( $>150 \mu \mathrm{m})$ especially in the cysts of spermatocytes (open arrows in Figures $2 \mathrm{~g}, \mathrm{~h}$ and $7 \mathrm{e}$ ). In the cysts of type $A$ or early type $B$ spermatogonia, testis-ova were smaller (diameter $<100 \mu \mathrm{m}$; arrows in Figures $2 \mathrm{~h}$ and $7 \mathrm{e}$ ) than those in the cysts of spermatocytes (open arrows in Figures $2 \mathrm{~g}, \mathrm{~h}$ and $7 \mathrm{e}$ ). Image analysis based on mathematical morphology confirmed the increase of the largely developed testis-ova (diameter $>150 \mu \mathrm{m}$ ) 7-14 days after irradiation (Figure 5). Moreover, the increased number of testis-ova continued up to 14 days after irradiation (Figure 3).

Twenty-eight days (1 month) after irradiation, almost all testis-ova disappeared from the testes (Figures $2 \mathrm{i}$ and j); hence, the restoration of spermatogenesis was clearly evident compared with non-irradiated testes (Figures 1d and f).

Morphological changes of wt Hd-rR spermatogenesis during 1 month after $\mathbf{5}$ Gy $\gamma$-ray irradiation. One day after $\gamma$-ray (5Gy) irradiation, strongly H\&E-stained pyknotic cells and anti-activated caspase- 3 immunostaining-positive cells appeared in the cysts of early type B spermatogonia (arrowheads in Figures 6a and c), indicating that the pyknotic cells were apoptotic. Almost all the pyknotic cells disappeared within 7 days after irradiation (Figures $6 d$ and e). The numbers of type B spermatogonia and spermatocyte cysts decreased, and the whole testis was filled with spermatids and spermatozoa (Figures $6 \mathrm{~d}$ and e); in addition, the Sertoli cells making the boundaries of the cysts were remarkably thicker (Figures $6 e$ and f). Electron microscopic observations revealed that in the enlarged Sertoli cells, many apoptotic 

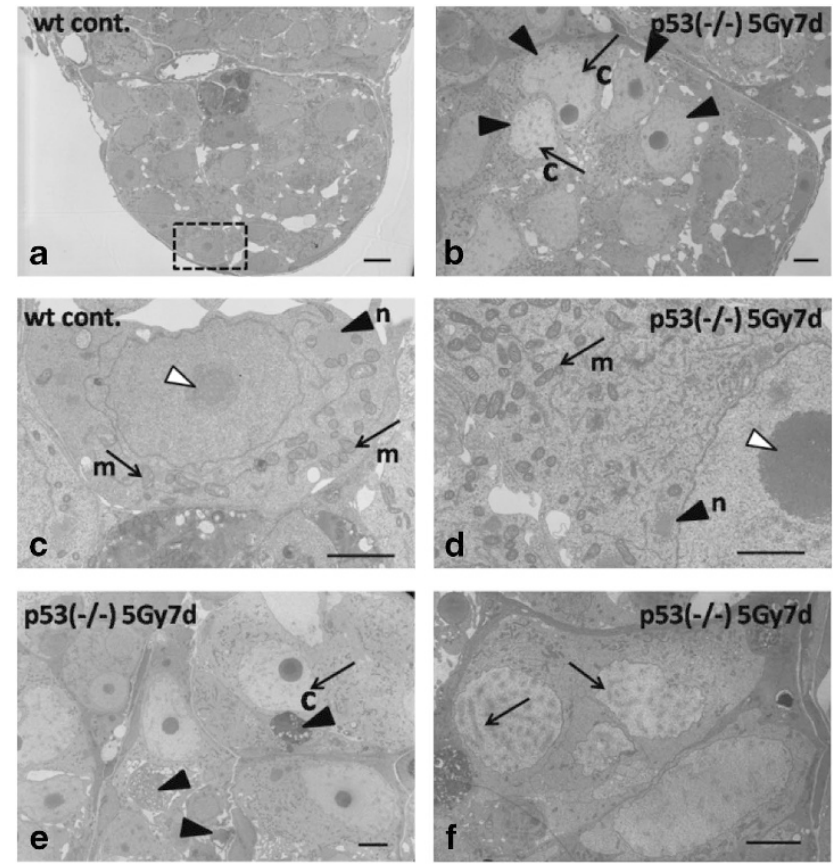

Figure 4 Electron microscopic observations of testis-ova in p53 $(-/-)$ mutants 7 days after irradiation with $\gamma$-rays (5 Gy). Morphological changes of testisova after $\gamma$-ray (5Gy) irradiation. (a) Electron microscopic observation of wt spermatogonia. (c) Enlarged view of the boxed area in (a) showing the germinal dense body (nuage) in the cytoplasm (arrowhead with $\mathrm{n}$ in c), which is closely associated with large aggregations of mitochondria (arrows with $\mathrm{m}$ in $\mathrm{c}$ ). The nucleolus of a spermatogonia is shown by an open arrowhead in c. (d) In the cytoplasm of p53( - / - ) testis-ova, larger and more electron-dense nucleoli (e.g., open arrowhead in $\mathbf{d}$ ) are present. It is noticeable that an extensive number of mitochondria and more electron-dense nuage are present (arrow with $\mathrm{m}$ and arrowhead with $n$ in $d$ ) compared with those of wt spermatogonia (arrows with $m$ and arrowhead with $\mathrm{n}$ in $\mathbf{c}$ ). (b) The testis-ova in the irradiated testis increased synchronously (arrowheads in $\mathbf{b}$ ) and they had a characteristic appearance of short and thick chromatin (arrows with $\mathbf{c}$ in $\mathbf{b}$ and e). (e) Apoptotic condensed nuclei were observed nearby testis-ova (arrowheads in e). (f) Some testis-ova have synaptonemal complexes in the nucleus (arrows in $\mathrm{f}$ ). Chromatin (c), mitochondria (m), nuage (n). Scale bars represent $2 \mu \mathrm{m}$ in $\mathbf{c}$ and $\mathbf{d} ; 5 \mu \mathrm{m}$ in $\mathbf{a}, \mathbf{b}, \mathbf{e}$ and $\mathbf{f}$

cells were phagocytosed and digested (arrowhead in Supplementary Figure S1A, B). The histological appearance of the irradiated testes at 14 days after irradiation was the same as those at 7 days after irradiation (Figures $6 \mathrm{~d}-\mathrm{h}$ ), and spermatogenesis was restored at 28 days ( 1 month) after irradiation (Figures $6 i$ and $j$ ). These histological changes of spermatogenesis in the $\gamma$-ray-irradiated testes are consistent with those reported in previous papers in which no testisovum was observed. ${ }^{10,11}$

\footnotetext{
Morphological changes of testis-ova in p53-deficient medaka mutants during 1 month after $1 \mathrm{~Gy} \gamma$-ray irradiation. One day after $1 \mathrm{~Gy} \gamma$-ray irradiation, there were no apparent histological changes (Figure 7a), as after 5 Gy irradiation (Figures $2 a$ and $b$ ). Seven days after irradiation, there were fewer testis-ova than in 5 Gy-irradiated testes (arrows in Figure 7c), and strongly H\&E-stained pyknotic cells appeared near the testis-ova (arrowheads in Figure 7c). Fourteen days after irradiation, the number of
}
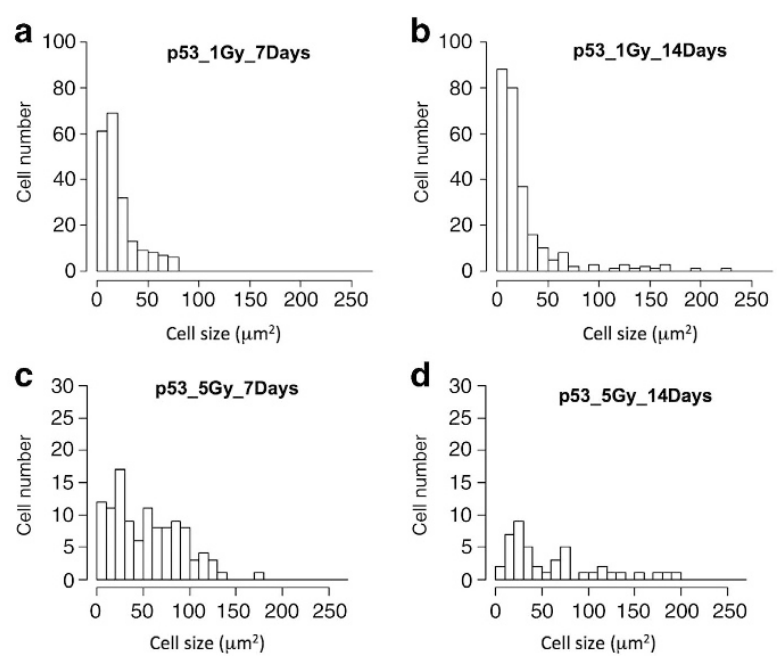

Figure 5 Histograms of type A spermatogonia and testis-ova in p53(-/ - ) testes 7 days and 14 days after irradiation. Areas of type A spermatogonia and testis-ova were segmented and extracted from the pictures of p53 $(-/-)$ testes after 7 days and 14 days after irradiation with $\gamma$-rays ( 1 and 5 Gy) by image processing procedures based on mathematical morphology. As type A spermatogonia and testis-ova were not able to be identified definitely by their size, we prepared the histograms for the cells including both type A spermatogonia and testis-ova, calculated by the mathematical morphology-based image processing method. (a) p53 $(-/-)$ testes after 7 days with $\gamma$-ray 1 Gy, (b) p53 $(-/-)$ testes after 14 days with $\gamma$-ray 1 Gy, (c) p53 $(-/-)$ testes after 7 days with $\gamma$-ray 5 Gy and (d) p53(-/-) testes after 14 days with $\gamma$-ray 5 Gy

testis-ova increased and their size was larger than those at 7 days after irradiation (Figures $5 \mathrm{a}, \mathrm{b}$ and $7 \mathrm{~b}-\mathrm{e}$ ). Twentyeight days after irradiation, testis-ova disappeared and normal spermatogenesis was restored (Figure 7f). These results indicated that the time courses of histological changes in testes during 1 month after 1 and 5 Gy $\gamma$-ray irradiation are almost the same, but the number and the size of testis-ova in the irradiated testes were dose-dependent.

Expressions of $425 p 50$, Oct 4 and vitellogenin genes were examined in irradiated p53-deficient medaka testes or liver. Gene expression of an ovary-specific marker, $42 \mathrm{Sp} 50,{ }^{20}$ was examined in non-irradiated and irradiated testes of wt Hd-rR and p53-deficient medaka males 7 days after 5 Gy $\gamma$-ray irradiation. As clearly shown in Figure $8 \mathrm{a}$, 42 Sp50 transcripts were amplified in non-irradiated and irradiated p53-deficient medaka testes, as well as in ovaries of wt and p53-deficient medaka. By ISH on sections of gonads of p53-deficient medaka at 7 days after the irradiation, 42Sp50 was expressed in the testis-ova (arrows in Figure 8c). By contrast, 42Sp50 was not expressed in irradiated or non-irradiated wt testes (data were not shown).

As $42 S p 50$ gene could only express in previtellogenic oocytes later than late pachytene in division I of meiosis, ${ }^{20}$ we examined the gene expression of Oct4, which would express in the primordial germ cells (PGCs) especially in ovary. ${ }^{21}$ Analysis using RT-PCR showed that Oct4 transcripts were amplified in non-irradiated and irradiated p53-deficient medaka testes 7 days after irradiation, as well as in ovaries of wt and p53-deficient medaka (Figure 8d). 

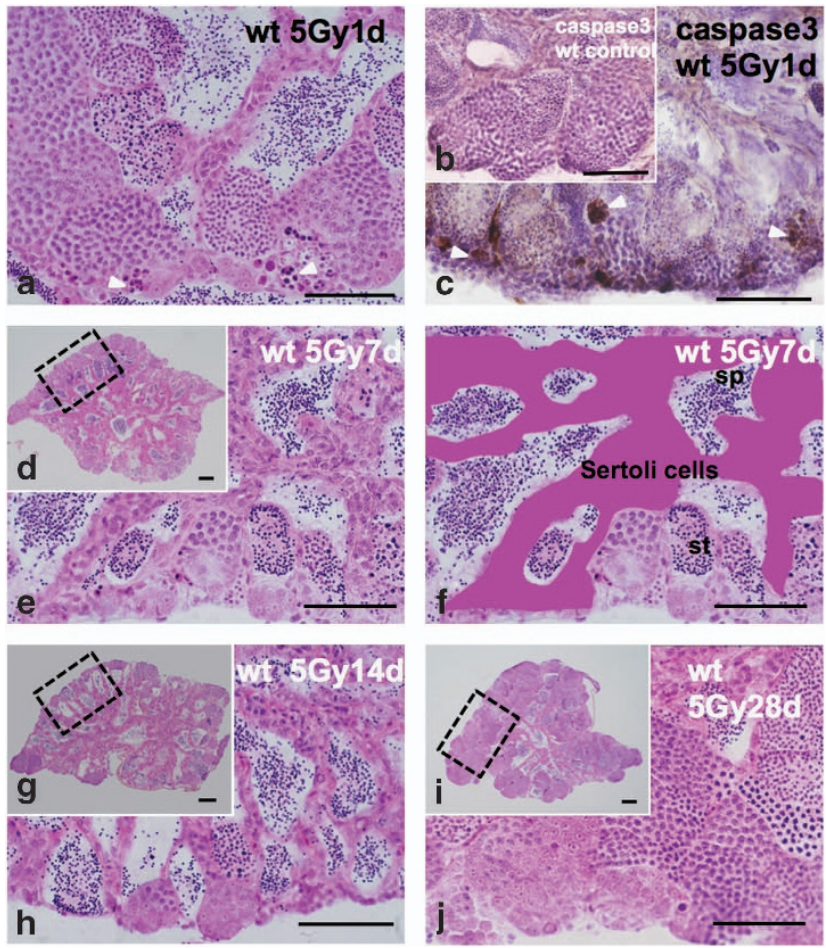

Figure 6 Histological changes of wt testes during 1 month after $\gamma$-ray (5Gy) irradiation. (a) H\&E-stained section of wt testis 1 day after irradiation. Many pyknotic cells were observed in the cysts of early type B spermatogonia (arrowheads in a). (c) Immunohistochemistry against anticleaved caspase3 showing the presence of immuno-positive cells in the cysts of early type B spermatogonia (arrowheads in c), where pyknotic cells (arrowheads in a) are positioned, in contrast to no positive signal shown in control wt medaka testis (b). (d) Histological H\&E-stained section of wt testis 7 days after irradiation, and (e) enlarged view of the boxed area in (d), showing the histological appearance of hypertrophied Sertoli cells. (f) A schematic representation of hypertrophied Sertoli cells (pink) of (e). (g) Histological H\&Estained section of wt testis 14 days after the irradiation, and (h) enlarged view of the boxed area in (g), showing the hypertrophied Sertoli cells. (i) Histological H\&Estained section of wt testis 28 days after irradiation and (j) enlarged view of the boxed area in (i), showing histological appearance of the clear restoration of spermatogenesis. Scale bars represent $50 \mu \mathrm{m}$

By contrast, Oct4 were not expressed in non-irradiated and irradiated wt testes (Figure 8d).

Moreover, to confirm whether the testis-ova could develop to vitellogenic oocytes (Stage VI) in irradiated p53-deficient medaka testes, we examined the gene expression of vitellogenin (VTG2), which is a commonly accepted biomarker of estrogenic activity. ${ }^{22}$ VTG2 is specifically synthesized in the liver of females, secreted into the blood stream and finally taken up by the oocytes. ${ }^{22}$ Analysis using RT-PCR showed that VTG2 transcripts in p53-deficient male medaka liver were never expressed in non-irradiated and irradiated 7 days and 14 days after 5 Gy $\gamma$-ray irradiation as well as in non-irradiated and irradiated wt male medaka liver (Figure 8e). By contrast, VTG2 were clearly expressed in wt and p53-deficient female medaka liver (Figure 8e).

These three kinds of gene analyses, therefore, demonstrated that oocyte-like cells found in non-irradiated and irradiated p53-deficient medaka testes were pre-vitellogenic oocytes, which never develop up to vitellogenic oocytes by IR irradiation.
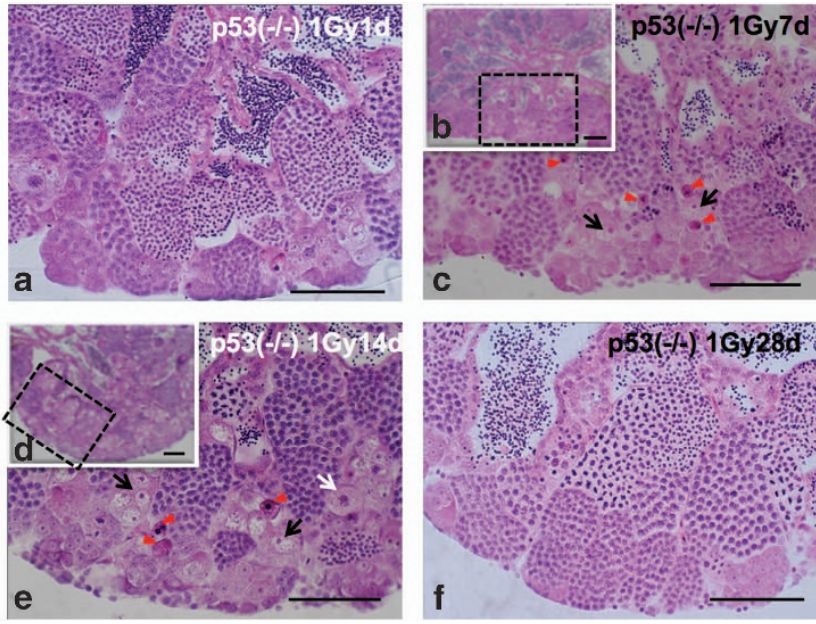

Figure 7 Histological changes of testis-ova in p53 $(-/-)$ testes during 1 month after $\gamma$-ray (1 Gy) irradiation. (a) H\&E-stained section of p53 ( - I-) testis 1 day after irradiation showing no apparent histological changes. (b) Histological H\&E-stained section of p53( $-I-)$ testis 7 days after irradiation and (c) enlarged view of the boxed area in B, showing pyknotic cells (arrowheads in $\mathbf{c}$ ) and increased testis-ova (arrows in c). (d) Histological H\&E-stained section of p53( - / - ) testis 14 days after irradiation and (e) enlarged view of the boxed area in (d), showing pyknotic cells (arrowheads in e) and testis-ova (arrows in e) were present. (f) Histological $\mathrm{H} \& \mathrm{E}$-stained section of $\mathrm{p} 53(-/-)$ testis 28 days after irradiation showing that spermatogenesis was restored almost completely, and that the number of testis-ova had decreased. Scale bars represent $50 \mu \mathrm{m}$
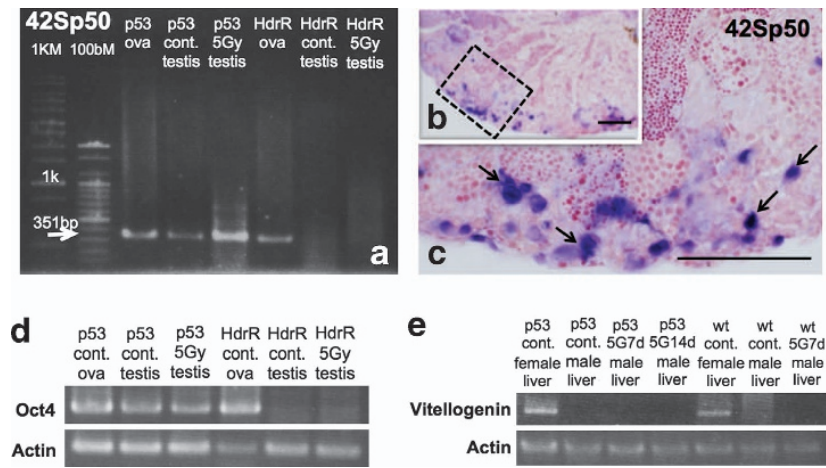

e

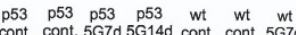
female male male male female male male
liver liver liver liver liver liver liver Actin

Figure 8 Gene expressions in p53 (- / - ) testes after $\gamma$-ray (5 Gy) irradiation. (a) Transcript of $42 \mathrm{Sp} 50$ were amplified both in non-irradiated and irradiated p53 $(-/-)$ testes with $\gamma$-rays (5Gy), as well as in p53 $(-/-)$ and wt Hd-rR ovaries, while not amplified in non-irradiated and irradiated wt testes. (b) Expression of $42 \mathrm{Sp} 50$ in irradiated p53 $(-/-)$ testes was investigated on histological sections by ISH and as clearly shown in the enlarged view (c) of the boxed area in $\mathbf{b}$, testisova in the p53 $(-/-)$-irradiated testes were positive (blue-stained cells; arrows in c). (d) Transcript of Oct4 were amplified both in non-irradiated and irradiated p53 $(-/-)$ testes with $\gamma$-rays (5 Gy), as well as in p53 $(-/-)$ and wt $\mathrm{Hd}-\mathrm{rR}$ ovaries, while not amplified in non-irradiated and irradiated wt testes. (e) Transcripts of vitellogenin were not amplified from male liver cDNA of wt and p53(-/ - ), without irradiation and with $\gamma$-ray (5 Gy) irradiation at 7 days and 14 days. However, they were clearly amplified from female liver of both control wt and p53 $(-/-)$. Scale bars represent $50 \mu \mathrm{m}$

\section{Discussion}

In this study, we found a small number of testis-ova in the testes of p53-deficient medaka, mainly in the cysts of type A or early type B spermatogonia. In addition, the testis-ova 
expressed the $42 S p 50$ and Oct4 genes, indicating that the oocyte-like cells found in p53-deficient medaka testes are derived from sexual trans-differentiation, which are never found in wt testes. Gamma-ray irradiation induced proliferation of testis-ova in p53-deficient medaka testes within 7 days after irradiation. Electron microscopic observations and RT-PCR analysis of vitellogenin demonstrated that the testis-ova proceeded up to the zygotene or pachytene stages of premature meiosis in the testis, and failed to mature beyond the pachytene stage, at which stage recombination between chromosomes occurs. Within 28 days after irradiation, almost all testis-ova disappeared by p53-independent apoptosis, and normal spermatogenesis was restored as in wt testis.

Adult germ cells of medaka testis can be bipotential and p53 is pivotal to suppress the differentiation of testisova in testis. Medaka have an $X X / X Y$ sex determination system like mammals, ${ }^{14-16}$ and $D M Y / d m r t 1 b Y$ has been identified as the dominant male-determining gene on the $Y$ chromosome, ${ }^{14,15}$ while many other teleosts such as zebrafish have no obvious heteromorphic sex chromosomes. ${ }^{23,24}$ In zebrafish, all embryos at first have undifferentiated ovary-like gonads regardless of their intrinsic sex, and all oocyte-like germ cells disappear by apoptosis in the gonad of male zebrafish. ${ }^{25,26}$ In this study, all p53-deficient male medaka are as fertile as wt male medaka and have a typical male-type external appearance such as dorsal and anal fins (Figures 1a and b), suggesting that the physiological functions of sex steroids such as estrogen or androgen in p53-deficient medaka are normal. Light and electron microscopic observations of the irradiated p53-deficient medaka testes revealed that all testis-ova start to proliferate synchronously in cysts filled with type A or early type B spermatogonia. These observations suggest that testis-ova are not produced as a result of disorder in the compartmentalization around the spermatogonia.

The diplotene stage is known to be the critical period for sex determination in zebrafish. In fancl (Fanconi anemia complementation group L) mutant zebrafish, abnormally increased apoptosis in oocytes at earlier stages than the diplotene stage induced female-to-male sex reversal. The introduction of the p53 mutation into the fancl mutant reduced germ cell apoptosis and rescued the fancl sex reversal phenotype. ${ }^{27}$ This report showed that the progress of meiosis to the diplotene stage is essential to feminize developing gonads in zebrafish embryos. In combination with medaka data, male germ cells with DNA damage may cause transdifferentiation into female-type meiosis, if they were not eliminated by apoptosis. In this study, electron microscopic observations showed that testis-ova would not enter into the diplotene stage in the irradiated p53-deficient medaka testes (Figure 4f). In addition, analysis using RT-PCR of VTG2 showed that testis-ova never proceeded up to vitellogenic oocytes in irradiated testes 7 days and 14 days after irradiation (Figure 8e). These suggest that to maintain the gonad as a testis, elimination of meiotic oocytes before they enter into the diplotene stage is essential, hence, elimination may also be necessary to suppress sex reversal from male to female in medaka. This is in contrast to the testes administrated hormonal treatment, which results in the presence of testis-ova at the diplotene stage. ${ }^{17}$

Although the medaka has an $X X / X Y$ sex chromosome system, ${ }^{14-16}$ environmental factors such as high temperature or sex steroids can induce female-to-male sex reversal at the embryonic stage. ${ }^{28,29}$ This suggests that medaka germ cells in developing gonads are sexually bipotential. Furthermore, in adult testis, testis-ova can be produced in the recovering process of spermatogenesis, which was impaired by administration of sex steroids; moreover, high temperature or starvation could promote the production of them. ${ }^{30,31}$ Administration of sex steroids is a prerequisite for the induction of testis-ova in adult testes: ${ }^{17,18,30,31}$ however, we have demonstrated that irradiation by $\gamma$-rays alone could induce the proliferation and development of testis-ova in the absence of p53 activity. Furthermore, testis-ova observed in the irradiated testes appeared much earlier, 3 days after irradiation compared with 2 weeks after hormonal treatment. ${ }^{17}$ Moreover, we examined the expression of Oct4 gene. ${ }^{22}$ Qin et al. ${ }^{32}$ reported that the expressions of oct4 gene in human embryonic stem cells at mRNA and protein level decreased after UV irradiation in contrast to p53si cells that were not downregulated by UV irradiation, demonstrating that the $p 53$ gene bind to the promoter region of Oct4. In this present study, the expressions of Oct4 gene by RT-PCR demonstrated that control p53-deficient male medaka have PGCs as well as those in ovary (Figure $8 \mathrm{e}$ ). These results strongly suggest that SSC keep sexual bipotentiality in the adult testis and in developing gonads, and that p53 has a critical role in the testis to suppress the bipotentiality of the SSC to differentiate into ova. As no testis-ova were found in the wt Hd-rR testes by histological analysis (Figures 1c and e) and Oct4 gene expression by RT-PCR (Figure 8e), testis of wt medaka could contain a small number of spontaneously differentiated testis-ova that might be normally eliminated by p53-dependent apoptosis.

Irradiation promotes premature meiosis and proliferation of testis-ova, and p53 regulates the cell cycle in spermatogenesis. Gamma-ray irradiation induced proliferation of testis-ova in p53-deficient medaka testes, and they proceeded up to the zygotene or pachytene stages of premature meiosis in the testis. A 5Gy and 1Gy $\gamma$-ray irradiation dose induced these sequential changes of testisova (Figures 2 and 7), although a 1 Gy dose is much lower than the lethal dose $\left(L D_{50}\right)$ for adult medaka. This result indicates that morphological changes of testis-ova are highly sensitive to IR irradiation. The number and the size of testisova that were irradiated at $1 \mathrm{~Gy}$ were lower and smaller than those irradiated at $5 \mathrm{~Gy}$ (Figure 5), indicating that morphological changes of irradiated testis-ova are dose-dependent.

In general, irradiation either activates checkpoints that lead to cell cycle arrest to make time to repair the genomic lesions or induces apoptosis to eliminate the damaged cells. ${ }^{33}$ Moreover, Kuwahara et al. reported the 'reset system' to efficiently remove damaged spermatogenic cells, except for SSC, by accelerating spermatogenesis. ${ }^{10,11}$ We hypothesized that accelerated spermatogenesis after irradiation would result in an increase of testis-ova in the cysts of p53-deficient medaka testes. On the other hand, it was 
reported that in rat meiotic cells, cell cycle arrest of G1 delay would not occur, whereas the cell cycle arrest occurred in type B spermatogonia in the mitotic $S$ phase. ${ }^{34}$ This report suggests another possibility that irradiated testis-ova could not activate the checkpoint system in the meiotic phase, which resulted the progression into the zygotene or pachytene of meiosis.

This study showed that p53-independent apoptosis could eliminate almost all of the abnormally increased meiotic testis-ova, even if the function of p53 to activate apoptosis induction was deficient. Electron microscopic observation showed that the apoptotic cells were phagocytosed in enlarged Sertoli cells in p53-deficient medaka testes (Supplementary Figure S1C-E). Sertoli cells are supporting somatic cells in the testis, enclosing the testicular germ cells; however, little is known about the interplay between Sertoli cells and germ cells. Our results demonstrated that Sertoli cells have a pivotal role in the removal of apoptotic cells by phagocytosis. This elimination system of immature meiotic testis-ova could restore spermatogenesis within 28 days after $\gamma$-ray irradiation.

In the embryonic gonads of medaka, granulosa cells in the ovary and Sertoli cells in the testis develop from the common precursor of the sox9b-expressing supporting cells. ${ }^{35}$ In the ovary, oocytes differentiated and proceeded to meiosis up to the early diplotene stage in a unique compartment of the ovary called the 'germinal cradle', ${ }^{36}$ which might be similar to the cyst compartments in the testes where spermatogenic cells are differentiating synchronously. In the germinal cradle of the ovary, some of the meiotic maturing oocytes undergo apoptosis in sox9b-expressing somatic cells, ${ }^{36}$ which was similar to our demonstration that apoptotic cells were phagocytosed in male Sertoli cells. These findings raised the possibility that adult ovaries and testes are composed of structurally similar units, which results in sexual bipotentiality toward both pathways as well as developing gonads.

For normal development of ova, it is essential that a reciprocal cross-talk between the germ cells and gonadal somatic cells is required for feminizing the gonads. ${ }^{8}$ This finding supports the possibility that irradiated testis-ova could not develop beyond the pachytene stage because there were no ovarian somatic cells to support differentiation and development of testis-ova in the irradiated p53-deficient medaka testes.

This study is the first report that the $p 53$ gene has a pivotal role in the elimination of the spontaneous testis-ova in testes. However, p53 is not indispensable for the restoration of spermatogenesis in impaired p53-deficient medaka testes in which abnormally increased premature meiotic testis-ova could not enter into the diplotene stage after irradiation. In the p53-knockout mouse testes, large-sized SSC lying at the basal membrane were also observed, and 10 days after a 5 Gy dose of X-rays, these large-sized spermatogonial cells increased. No significant increase in the number of the large spermatogonia was seen in the heterozygotes. ${ }^{37}$ These cells may be produced by similar mechanisms as the medaka testis-ova after irradiation, although sex determination in mammals is less plastic or labile than fish. Therefore, the medaka p53 mutant will be a good model for understanding trans-differentiation of gametes in vertebrates.

\section{Materials and Methods}

Medaka. The p53-deficient fishes were originally generated by TILLING. ${ }^{4}$ In this study, these fish were backcrossed four times with inbred $\mathrm{Hd}$-rR fishes to establish a p53-deficient strain with a Hd-rR genomic background. The fishes were kept at room temperature $\left(26-28^{\circ} \mathrm{C}\right)$ under a 14-h light and 10-h dark cycle, fed on powdered diet (Tetra-min, Tetra Werke Co., Mells, Germany) and brine shrimp (Artemia franciscana) three times a day. This study was conducted according to 'The University of Tokyo Animal Experiment Enforcement Rule'.

Irradiation. Sexually mature, 1-year-old male fish were subjected to wholebody irradiation from ${ }^{137} \mathrm{Cs} \gamma$-rays (Gammacell 3000Elan, MDS Nordion, Ottawa, ON, Canada) with a dose rate of $10 \mathrm{~Gy} / \mathrm{min}$ at room temperature in a watercontaining plastic tube without anesthesia at doses of 1 and 5 Gy.

Histology. For light microscopic observations, testes were isolated, fixed in Davidson fixative solution overnight at $4^{\circ} \mathrm{C}$, washed in PBS $(-)$, dehydrated with ethanol and embedded in plastic resin (Technovit 8100, Heraeus Kulzer, Wehrheim, Germany). We prepared 8- $\mu$ m-thick sections of the central part of the testes from wt $\mathrm{Hd}-\mathrm{rR}$ and p53-deficient medaka fish. Sections were stained with H\&E. For immunostaining using polyclonal anticleaved caspase 3 antibodies (9661S, Cell Signalling Technology, Beverly, MA, USA) (1:200), isolated testes were fixed in $4 \%$ paraformaldehyde in $0.1 \mathrm{M} \mathrm{PB}$ overnight at $4^{\circ} \mathrm{C}$. Cryostat sectioning and immunostaining were performed as described previously, ${ }^{38}$ then developed in $0.05 \%$ diaminobenzidine, counterstained with hematoxylin and photographed with an Olympus BX50 (Tokyo, Japan) microscope equipped with a DP70 Olympus digital camera.

For electron microscopic observations, the testes was isolated and prepared as described previously. ${ }^{39}$ Ultrathin sections were cut, stained with uranyl acetate and lead citrate, and samples were examined with a Hitachi H-7500 electron microscope (Tokyo, Japan) operated at $80 \mathrm{kV}$.

Quantification of the number and the size of testis-ova. We counted the number of testis-ova on histological sections in non-irradiated wt $\mathrm{Hd}$ $\mathrm{rR}$ and p53-deficient medaka testes $(n=3)$, and $\gamma$-ray-irradiated p53-deficient medaka testes at 1 day, 7 days, 14 days and 28 days after irradiation (all time points, $n=3$ ), followed by dividing each total area of testes calculated by Image $\mathrm{J}$ software (NIH, Bethesda, MD, USA). Moreover, we counted the number of spermatocytes in p53-deficient medaka testes of non-irradiated and $\gamma$-ray irradiated at 7 days and 14 days after irradiation (all time points, $n=1$ ).

In this paper, the mathematical morphology-based image processing method was used for cell segmentation in the images of the histological sections. ${ }^{40}$ Mathematical morphology is suitable for handling shape-related processing. The segmentation approach was processed as follows with four steps. First, the regions of target cells (i.e. spermatogonia and testis-ova) were extracted by the top-hat filter based on rotational morphological processing. ${ }^{41}$ The filter extracts these cell regions with sizes corresponding to the size of the used structuring element, which is a small image used for morphological operations. The size was empirically determined. Second, the extracted regions were binarized by an automatic thresholding technique. Third, small isolated pixel regions, which can be considered as segmentation noise, were eliminated by the opening operation. Fourth, the area of the cell regions was calculated. These processing were performed using $R$, an open source language for statistical computing, data analysis and graphical visualization.

RT-PCR and ISH. Total RNA was extracted from wt and p53-deficient medaka adult dissected testes or liver. cDNAs were synthesized from $1 \mu \mathrm{g}$ of total RNA from medaka testis or liver using ReverTra Ace- $\alpha$ (Toyobo, Osaka, Japan) and oligo(dT)20 primers. ${ }^{42}$ Equal amounts of first-strand cDNAs were then amplified using primer pairs for the studied genes as follows: 42Sp50 forward 5'-CAACGTGGTCGTCATCGGTC-3'; 42 Sp50 reverse 5'-GGCTTCATACTCCCC TTTGG-3'; 20 Oct4 forward $5^{\prime}$-GGCGTAAACTCGTCTCAAGG-3'; Oct 4 reverse 5'-CCAAACCCAGATCGTCTGAT-3'; VTG2 forward 5'-CATGCTAAACGAGCT GACCA-3'; VTG2 reverse 5'-TTGACCAAGATGCACTGAGC-3'; Actin forward 5'-CACTCTGAGCGCCGTCACACACAG-3'; Actin reverse 5'-TGACACCCTGGTG CCTGGGGCGAC- $3^{\prime}$. The PCR reactions were performed using 40 cycles of $95^{\circ} \mathrm{C}$ for $30 \mathrm{~s}, 60^{\circ} \mathrm{C}$ for $30 \mathrm{~s}$ and $72{ }^{\circ} \mathrm{C}$ for $1 \mathrm{~min}$.

42 Sp50 cDNA was cloned in pCR4-TOPO using DNA fragment of 42 Sp50 transcript being amplified and probe synthesis was produced by digesting plasmids with Not1 and transcribing with T3 polymerase for ISH. The isolated testes were cut into 3-4 pieces, and subjected to whole-mount $\mathrm{ISH}^{43}$ The specimens were 
embedded in Technovit 8100, sectioned and counterstained with $0.5 \%$ neutral red (Muto Pure Chemicals, Tokyo, Japan).

\section{Conflict of Interest}

The authors declare no conflict of interest.

Acknowledgements. We thank Dr Yoshio Sekiya, Mr Toshikazu Yamashita, Mrs Toyoko Kuno, Mr Takuya Kobayashi and Mr Yusuke Hibi for their assistance in establishing and maintaining the p53 mutant strain with the inbred $\mathrm{Hd}-\mathrm{rR}$ background. Mathematical morphology-based image processing for the image segmentation was supported by the Imaging Science Program of NINS. Special thanks to Dr Hiroshi Sagara, Dr Haruo Mizutani, Dr Aiko Hirata and Mrs Sawako Niki for their technical help and helpful advice about electron microscopic observations. This work was supported by Individual Collaborative Research Program (12-347) from National Institute for Basic Biology to SO. This research was partly supported by a Grant-in-Aid for Scientific Research to HM (21221003) from the Ministry of Education, Culture, Sports, Science and Technology of Japan, and by the FY2006 Ground-based Research Program for Space Utilization Research from the Japan Space Forum (to HM).

1. Shima $A$, Shimada $A$. The medaka as a model for studying germ-cell mutagenesis and genomic instability. Mar Biotechnol 2001; 3: 162-167.

2. Ishikawa Y, Hyodo-Taguchi Y. Heritable malformations in the progeny of the male medaka (Oryzias latipes) irradiated with X-rays. Mutat Res 1997; 389: 149-155.

3. Norimura T, Nomoto S, Katsuki M, Gondo Y, Kondo S. p53-dependent apoptosis suppresses radiation-induced teratogenesis. Nat Med 1996; 2: 577-580.

4. Taniguchi Y, Takeda S, Furutani-Seiki M, Kamei Y, Todo T, Sasado T et al. Generation of medaka gene knockout models by target-selected mutagenesis. Genome Biol 2006; 7: R116.

5. Shima A, Shimada A. Induction of mutations in males of the fish Oryzias latipes at a specific locus after gamma-irradiation. Mutat Res 1988; 198: 93-98.

6. Shima A, Shimada A. Development of a possible nonmammalian test system for radiationinduced germ-cell mutagenesis using a fish, the Japanese medaka (Oryzias-latipes). Proc Natl Acad Sci USA 1991; 88: 2545-2549.

7. Kanamori A, Nagahama Y, Egami N. Development of the tissue architecture in the gonads of the medaka Oryzias-Latipes. Zool Sci 1985; 2: 695-706.

8. Kurokawa H, Saito D, Nakamura S, Katoh-Fukui Y, Ohta K, Baba T et al. Germ cells are essential for sexual dimorphism in the medaka gonad. Proc Natl Acad Sci USA 2007; 104: 16958-16963.

9. Grier HJ, Linton JR, Leatherland JF, Devlaming VL. Structural evidence for 2 different testicular types in teleost fishes. Am J Anat 1980; 159: 331-345.

10. Kuwahara Y, Shimada A, Mitani H, Shima A. A critical stage in spermatogenesis for radiation-induced cell death in the medaka fish, Oryzias latipes. Radiat Res 2002; 157: 386-392.

11. Kuwahara $Y$, Shimada A, Mitani H, Shima A. Gamma-ray exposure accelerates spermatogenesis of medaka fish, Oryzias latipes. Mol Reprod Dev 2003; 65: 204-211.

12. Erickson GF. Spontaneous sex reversal in crgan-cultures of embryonic male gonad of bird. J Embryol Exp Morph 1974; 31: 611-620.

13. Wallace H, Badawy GMI, Wallace BMN. Amphibian sex determination and sex reversal. Cell Mol Life Sci 1999; 55: 901-909.

14. Matsuda M, Nagahama Y, Shinomiya A, Sato T, Matsuda C, Kobayashi T et al. DMY is a $Y$-specific DM-domain gene required for male development in the medaka fish. Nature 2002; 417: 559-563.

15. Nanda I, Kondo M, Hornung U, Asakawa S, Winkler C, Shimizu A et al. A duplicated copy of DMRT1 in the sex-determining region of the $Y$ chromosome of the medaka, Oryzias latipes. Proc Natl Acad Sci USA 2002; 99: 11778-11783.

16. Saito D, Tanaka M. Comparative aspects of gonadal sex differentiation in medaka: a conserved role of developing oocytes in sexual canalization. Sex Dev 2009; 3: 99-107.

17. Shibata N, Hamaguchi S. Evidence for the sexual bipotentiality of spermatogonia in the fish, Oryzias latipes. J Exp Zool 1988; 245: 71-77.

18. Egami N. Production of testis-ova in adult males of Oryzias latipes. IV. Effect of X-ray irradiation on testis-ovum production. J Fac Sci Tokyo Univ 1955; 7: 429-441.
19. Satoh N. An ultrastructural study of sex differentiation in teleost Oryzias latipes. J Embryol Exp Morph 1974; 32: 195-215.

20. Kinoshita M, Okamoto G, Hirata T, Shinomiya A, Kobayashi T, Kubo Y et al. Transgenic medaka enables easy oocytes detection in live fish. Mol Reprod Dev 2009; 76: 202-207.

21. Sanchez-Sanchez AV, Camp E, Garcia-Espana A, Leal-Tassias A, Mullor JL. Medaka Oct4 is expressed during early embryo development, and in primordial germ cells and adult gonads. Dev Dynam 2010; 239: 672-679.

22. Islinger M, Yuan H, Voelkl A, Braunbeck T. Measurement of vitellogenin gene expression by RT-PCR as a tool to identify endocrine disruption in Japanese medaka (Oryzias latipes). Biomarkers 2002; 7: 80-93.

23. Schreeb KH, Groth G, Sachsse W, Freundt KJ. The karyotype of the zebrafish (Brachydanio-Rerio). J Exp Anim Sci 1993; 36: 27-31.

24. Pijnacker LP, Ferwerda MA. Zebrafish chromosome-banding. Genome 1995; 38: 1052-1055.

25. Uchida D, Yamashita M, Kitano T, Iguchi T. Oocyte apoptosis during the transition from ovary-like tissue to testes during sex differentiation of juvenile zebrafish. J Exp Biol 2002; 205: 711-718.

26. Maack G, Segner H. Morphological development of the gonads in zebrafish. J Fish Biol 2003; 62: 895-906.

27. Rodriguez-Mari A, Canestro C, BreMiller RA, Nguyen-Johnson A, Asakawa K, Kawakami K et al. Sex reversal in zebrafish fancl mutants is caused by Tp53-mediated germ cell apoptosis. Plos Genet 2010; 6: e1001034.

28. Sato T, Endo T, Yamahira K, Hamaguchi S, Sakaizumi M. Induction of female-to-male sex reversal by high temperature treatment in medaka, Oryzias latipes. Zool Sci 2005; 22: 985-988.

29. Yamamoto T. Artificial induction of functional sex-reversal in genotypic females of the medaka (Oryzias latipes). J Exp Zool 1958; 137: 227-264.

30. Egami N. Production of testis-ova in adult males of Oryzias latipes. VI. Effect on testisovum production of exposure to high temperature. Annot Zool Jpn 1955; 29: 11-18.

31. Egami N. Production of testis-ova in adult males of Oryzias latipes. III. Testis-ovum production in starved males. J Fac Sci Tokyo Univ 1955; 7: 421-428.

32. Qin H, Yu T, Qing T, Liu Y, Zhao Y, Cai J et al. Regulation of apoptosis and differentiation by p53 in human embryonic stem cells. J Biol Chem 2007; 282: 5842-5852.

33. Kaufmann WK, Paules RS. DNA damage and cell cycle checkpoints. Faseb J 1996; 10: 238-247.

34. West A, Lahdetie J. X-irradiation-induced changes in the progression of type B spermatogonia and preleptotene spermatocytes. Mol Reprod Dev 2001; 58: 78-87.

35. Nakamura S, Aoki Y, Saito D, Kuroki Y, Fujiyama A, Naruse K et al. Sox9b/sox9a2-EGFP transgenic medaka reveals the morphological reorganization of the gonads and a common precursor of both the female and male supporting cells. Mol Reprod Dev 2008; 75: 472-476.

36. Nakamura S, Kobayashi K, Nishimura T, Higashijima S, Tanaka M. Identification of germline stem cells in the ovary of the teleost medaka. Science 2010; 328: 1561-1563.

37. Beumer TL, Roepers-Gajadien HL, Gademan IS, van Buul PPW, Gil-Gomez G, Rutgers $\mathrm{DH}$ et al. The role of the tumor suppressor p53 in spermatogenesis. Cell Death Differ 1998; 5: 669-677.

38. Yasuda T, Oda S, Ishikawa Y, Watanabe-Asaka T, Hidaka M, Yasuda H et al. Live imaging of radiation-induced apoptosis by yolk injection of acridine orange in the developing optic tectum of medaka. J Radiat Res 2009; 50: 487-494.

39. Yasuda T, Yoshimoto M, Maeda K, Matsumoto A, Maruyama K, Ishikawa Y. Rapid and simple method for quantitative evaluation of neurocytotoxic effects of radiation on developing medaka brain. J Radiat Res 2008; 49: 533-540.

40. Haralick RM, Sternberg SR, Zhuang XH. Image-analysis using mathematical morphology. IEEE T Pattern Anal 1987; 9: 532-550.

41. Kimori $Y$, Baba N, Morone N. Extended morphological processing: a practical method for automatic spot detection of biological markers from microscopic images. BMC Bioinform 2010; $11: 373$.

42. Hanno K, Oda S, Mitani H. Effects of dioxin isomers on induction of AhRs and CYP1A1 in early developmental stage embryos of medaka (Oryzias latipes). Chemosphere 2010; 78 : $830-839$.

43. Nemoto Y, Higuchi K, Baba O, Kudo A, Takano Y. Multinucleate osteoclasts in medaka as evidence of active bone remodeling. Bone 2007; 40: 399-408.

Cell Death and Disease is an open-access journal published by Nature Publishing Group. This work is licensed under the Creative Commons Attribution-NonCommercial-No Derivative Works 3.0 Unported License. To view a copy of this license, visit http://creativecommons.org/licenses/by-nc-nd/3.0/

Supplementary Information accompanies the paper on Cell Death and Disease website (http://www.nature.com/cddis) 